\title{
AC 2011-2848: CROSS-DISCIPLINARY BIOMEDICAL ENGINEERING LABORATORIES AND ASSESSMENT OF THEIR IMPACT ON STUDENT LEARNING
}

\author{
John D. Gassert, Milwaukee School of Engineering
}

JOHN D. GASSERT, Ph.D., P.E.

John D. Gassert is currently a Professor of Biomedical Engineering at the Milwaukee School of Engineering (MSOE) and an Adjunct Professor of Biophysics at the Medical College of Wisconsin. Gassert is a Registered Professional Engineer in the State of Wisconsin. He is currently the Chair of the Accreditation Activities Committee for BMES. He earned his Ph.D. in Biomedical Engineering from Marquette University in 1995. He earned his MS degree and BE degree in Electrical Engineering in 1974 and 1971 respectively, both from Marquette University. Gassert is an AIMBE Fellow, a Senior Member of the IEEE, and a member in BMES and Sigma Xi. He is an ABET EAC program evaluator for Biomedical Engineering. He has developed and taught courses at both the graduate and undergraduate level in Biomedical Engineering, Medical Informatics, Perfusion, Electrical Engineering, Computer Engineering, and Electrical Engineering Technology. Prior to arriving at MSOE, Gassert spent seventeen years in industry in positions as a design engineer, a clinical engineer and a consultant. As part of his 17 years in industry, he owned his own engineering design and consulting company for eight years. He continues to operate his consulting and design business on a part-time basis.

Dr. Jeffrey A. LaMack, Milwaukee School of Engineering

Olga Imas, Milwaukee School of Engineering

Olga Imas, $\mathrm{PhD}$ is an assistant professor of biomedical engineering at the Milwaukee School of Engineering, where she teaches a variety of courses in biomedical digital signal processing, medical imaging, computing in biomedical engineering, biomaterials, anatomy and physiology. In addition to her academic responsibilities, she acts as a consultant to GE Healthcare for product development with emphasis on advanced imaging applications for neurology, cardiology, and oncology. Olga's technical areas of expertise include signal and imaging processing, and statistical analysis. In her previous and current product development roles, Olga gained extensive experience in clinical product management involving market analysis for new and existing imaging products, and clinical product marketing. She has experience in managing product evaluations at multiple clinical sites, and has a comprehensive knowledge of neurology, oncology, and cardiology imaging markets. She has established a number of strong collaborations with clinical experts in recognized neuroimaging and oncology centers.

Olga has earned her undergraduate degree in biomedical engineering from the Milwaukee School of Engineering in 1999, and a doctorate degree in biomedical engineering and functional imaging from the Joint Functional Imaging program at Marquette University and Medical College of Wisconsin in 2004. Prior to entering academia full-time in 2009, Olga completed a three-year postdoctoral fellowship in anesthesiology at the Medical College of Wisconsin, where she studied the effects of general anesthetic agents on brain function. She then worked at GE Healthcare as a product development specialist in CT and Molecular Imaging with emphasis on post-processing software applications for neurology, oncology, and cardiology. Olga has over twenty peer-reviewed publications, and three pending patents. Her professional interests include physiological mechanisms of Alzheimer's disease, anesthetic ablation of consciousness, and applicability of medical imaging in stroke and brain trauma.

Larry Fennigkoh, Milwaukee School of Engineering

NE Schlick, Milwaukee School of Engineering

Charles S. Tritt, Ph.D., Milwaukee School of Engineering

Dr. Tritt is an Associate Professor and the director of the Biomedical Engineering program at the Milwaukee School of Engineering. His professional interests include biomedical applications of mass, heat and momentum transfer; biomaterials, medical device and process modeling; medical device design; the FDA Quality System (also known as the current GMP) regulation (particularly design controls); and entrepreneurship.

Ron Gerrits, Milwaukee School of Engineering

(c)American Society for Engineering Education, 2011 
Ron J. Gerrits, Ph.D.

Ron Gerrits is currently an Associate Professor of Biomedical Engineering, and Academic Program Director of the Master's of Science in Perfusion program at the Milwaukee School of Engineering. He holds a B.S. in Biomedical Engineering from the Milwaukee School of Engineering (1994) and a Ph.D. in Physiology from the Medical College of Wisconsin (1999). He most commonly teaches health science courses for nursing, perfusion and biomedical engineering students. Professional interests focus on science education and he currently serves as the chairperson of the Curriculum and Instruction Committee of the Human Anatomy and Physiology Society and is active in an NSF funded Biology Scholars program 
Cross-Disciplinary Biomedical Engineering Laboratories and Assessment of their Impact on Student Learning

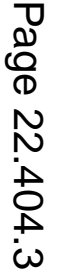




\begin{abstract}
Three cross-disciplinary team-based laboratory courses were introduced into the biomedical engineering curriculum at Milwaukee School of Engineering to enhance student understanding of the interdependence of the engineering topics and biomedical science. A major challenge for the faculty was the assessment of student understanding and retention of the materials, and judging if there has been an improvement as a result in the change in the curriculum. An established concept inventory was used to assess whether student competency in signals and systems was maintained in the new curriculum, despite a reduction in lecture hours in this core discipline to accommodate a concurrent cross-disciplinary laboratory with physiology. Students from both the prior and the new curricula were assessed. Appropriate statistical tests suggest that both student groups performed similarly on all questions. An average score of $52.1 \pm 27 \%$ (sd) was found for students associated with the curriculum without the cross-disciplinary labs. Students taking the cross-disciplinary course with the reduced lecture hours in biosystems had an average score of $46.7 \pm 28 \%$ (sd). The difference in performance between the two groups was not significant $(\mathrm{p}=0.50)$. However, anecdotal comments from students suggest some enhancement in the students' ability to solve multi-disciplinary problems. Future work will further assess the effectiveness of the new approach by testing student knowledge in the other core discipline, physiology, and in the integration of the multiple disciplines.
\end{abstract}

\title{
Introduction
}

In addition to the engineering sciences, every graduate of an engineering program is expected to learn professional skills such as teamwork, leadership, and communication. Biomedical Engineering (BME) programs must also provide the education that teach students the ability "to solve the problems at the interface of engineering and biology" and "the ability to make measurements on and interpret data from living systems."[1] Therefore, a good biomedical engineer must have a solid understanding of mechanical, electrical, and computer engineering fields, as well as biology, chemistry, and physiology, and must be able to combine and apply engineering and life science concepts to solve interdisciplinary problems in biomedical engineering.

In the course of its continuous improvement process, the BME faculty at the Milwaukee School of Engineering (MSOE) redesigned its curriculum to introduce more team-based interdisciplinary learning. The faculty members believe the most innovative additions to the curriculum are the three cross-disciplinary laboratory courses where students have to bring knowledge from (at least) two courses and solve problems at the interface of those disciplines. The decision to include these laboratory courses was in part based on the Engineer of 2020 report that recommended both team based and interdisciplinary learning [2]. The biomedical engineering faculty believes the new laboratory exercises will enhance student understanding of the interdependence of the engineering topics and biomedical science. At the same time, the faculty recognizes that it is equally important to not sacrifice student learning in the core disciplines with the implementation of the cross-disciplinary approach.

This paper will present an example of one of the cross-disciplinary laboratory courses. It will also examine an assessment tool that was used to evaluate whether conceptual learning in a core 
discipline was maintained when the new cross-disciplinary approach was used. Two groups of students, one taught in a curriculum without the cross-disciplinary laboratory courses and the other group taught using cross-disciplinary laboratory courses, were assessed using the tool, and the results are presented here. The assessment process was submitted to and approved by the MSOE Institutional Review Board (IRB).

\section{Description of Cross-Disciplinary Laboratory Courses}

In addition to many other courses with a laboratory component, the MSOE BME curriculum includes three cross-disciplinary laboratory courses. The first laboratory course is a Physiology/Biosystems Laboratory in which students are expected to take the concepts learned in the field of signals and systems and in physiology, and apply these concepts to address various experimental problems at the intersection of both fields. This course contains system modeling and analysis of physiological signals and systems.

The second laboratory course is a Physiology/Biofluids Laboratory. In this course, the students must apply the concepts from fluid dynamics and heat and mass transfer to physiological systems. Additional engineering tools, including model fitting and numerical simulation, are introduced in this course, adding further breadth.

The third cross-disciplinary laboratory course is a Biomechanics/Biomaterials Laboratory. This is the most interdisciplinary of the three courses, as the students have to bring together concepts learned in physics, chemistry, statics, dynamics, strength of materials, statistics, and biological systems to design and test implant materials and perform human measurement studies.

The previous curriculum featured the same number of laboratories as the new curriculum. However, the laboratories were associated with specific courses in that curriculum. To accommodate the cross-disciplinary laboratory courses in the new curriculum, it was necessary to eliminate one credit hour of lecture in each term from one of the courses in a core discipline. Despite the reduction in lecture hours, it was hypothesized that the student comprehension of all fundamental concepts in that discipline would be maintained at the level equivalent to that of the old curriculum, since the material was being concurrently applied in a cross-disciplinary laboratory.

\section{Example of a Cross-Disciplinary Laboratory Module}

It is expected that these three laboratory courses will help students learn that the term "interdisciplinary" refers not only to combining concepts from different disciplines, but to combining them is such a way that allows prediction of responses of complex systems. These courses are expected to improve the student's ability to think on a system level.

The following is an example from the Physiology/Biosystems laboratory where students were required to examine properties of a physiological system and methods used to model pressureflow relationships in the cardiovascular system. The students were expected to estimate the resistive and storage properties of the human circulatory system and then use 
MATLAB/Simulink and Multisim to study the transfer characteristics of an analog of the human vascular system.

The students were given background on a three-element and a four-element lumped parameter windkessel model of the human circulation as shown in Figure 1. They were also given estimated values for the various elements. The assignment was to develop a transfer function for the model based on these estimated values. The students were then expected to select an appropriate source that would represent the left ventricular output pressure and use that source as the input to each of the two windkessel models.

A

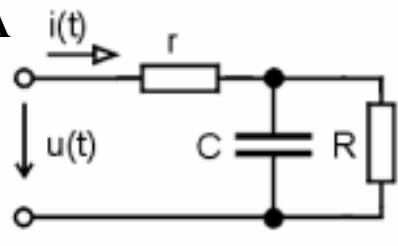

B

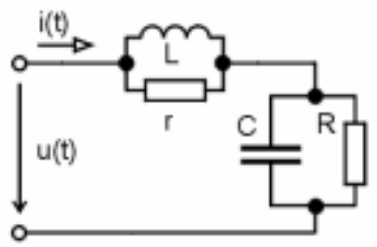

Figure 1: Schematic of a three-element (panel A) and a four-element (panel B) windkessel model of the human vascular system.

The deliverables for the exercise required that the students develop a hypothesis about the system response to their chosen input and test that hypothesis using Multisim to simulate the windkessel model response to a $1.0 \mathrm{~Hz}$ input signal. They were expected to determine a transfer function of the system using the values of the electrical analog components for the windkessel model. They scaled the magnitude of the input, $u(t)$, to a physiologically reasonable pressure pulse and verified these results using MATLAB. All results and an appropriate summary/concluding paragraph were documented in their logbooks.

In a later continuation of this study, students were asked to perform a frequency analysis of their own pulse plethysmogram and compare those results to the frequency analysis of the response from their windkessel model. These analyses were done in conjunction with a course that included coverage of cardiovascular physiology.

\section{Assessment Methods}

A major challenge for faculty is the assessment of student understanding and retention of the materials and evaluation of whether a curriculum change improved either understanding or retention $[3,4]$. With the above mentioned curriculum changes, the faculty wished to assess student learning in both the two core disciplines and at their intersection corresponding to a given cross-disciplinary laboratory. The first such laboratory course, combining physiology and biosystems, is the target for assessment in this study. For this pairing, the laboratory was detached from the physiology course in the new curriculum, and the number of lecture hours in the biosystems course was reduced from four to three.

The method chosen by the faculty was to, whenever possible, employ previously developed concept inventory (CI) assessment tools to assess students from both the prior and the new curriculum, and to draw inference from the results. A thorough literature review revealed a limited number of CI assessment tools and none were found for quantitative physiology and for 
cross-disciplinary disciplines such as physiology/biosystems. As a result, the decision was made to limit this study to the assessment of student comprehension of the core concepts in signals and systems using the concept inventory (SSCI) developed by Wage, et al. [5]. Specifically, the study tested the hypothesis that student comprehension of the core signals and systems concepts in the new curriculum is equivalent to that of the old curriculum. It was expected that the SSCI would provide a reasonable assessment of the students' ability to apply concepts of signals and systems. The SSCI exam consisted of 25 questions covering many fundamental concepts in signals and systems. An example of an SSCI question is seen in Figure 2.

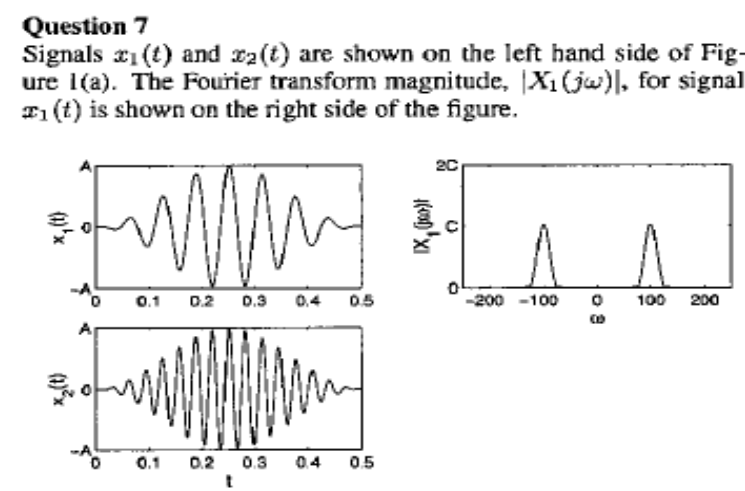

(a) Signals $x_{1}(t)$ and $x_{2}(t)$ and the Fourier transform magnitude $\left|X_{1}(j \omega)\right|$ for Question 7 .

Which of the plots shown in Figure 1(b) could be $\left|X_{2}(j \omega)\right|$, the Fourier transform magnitude for signal $x_{2}(t)$ ?
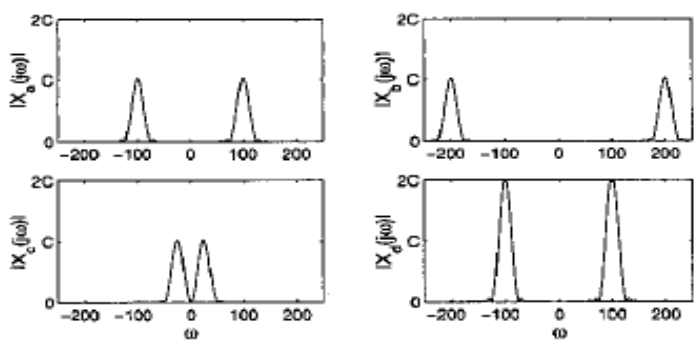

(b) Fourier transform magnitudes $\left|X_{\alpha}(j \omega)\right|$ through $\left|X_{d}(j \omega)\right|$ for Question 7.

Figure 2: An example of the questions used on the signals and systems concept inventory assessment test [5].

Students from curricula with $(n=28)$ and without $(n=26)$ the cross-disciplinary laboratory courses were tested to compare their understanding of signals and systems. Although not ideal, senior BME students from the curriculum that did not include cross-disciplinary laboratories were asked to take the SSCI assessment exam at the beginning of their senior year. These students had completed the second signals and systems course during the fall quarter in the prior academic year. As a result, some of the concepts had not been used academically for almost a year. The same SSCI assessment exam was also administered to junior BME students from the curriculum that included cross-disciplinary laboratory courses. This time the SSCI assessment exam was administered in the middle of the academic junior year. Overall SSCI performance was compared for the two groups using a Student's t-test. 


\section{Assessment Results}

Figure 3 shows the results of SSCI testing for both student groups on all 25 questions. Each bar represents the SSCI score computed as the percentage of students who responded correctly to a given question. The figure shows that both student groups performed similarly on all questions. The average SSCI score of $52.1 \pm 27 \%$ (sd) was found for students associated with the curriculum without the cross-disciplinary labs. Students taking the cross-disciplinary course with the reduced lecture hours in Biosystems had an average SSCI score of $46.7 \pm 28 \%$. These results are shown graphically in Figure 4. The difference in performance between the two groups was not significant $(\mathrm{p}=0.50)$. Overall, the results compare very favorably to "post-test" results reported by Buck et al. for electrical engineering students immediately following their signals and systems course [6]. Those scores ranged between 50 and 60 percent in that study.

It should be noted that in case of a lack of understanding of a concept underlying a given question, one would expect a SSCI score of $25 \%$, reflecting a random guess of the correct answer. However, the SSCI scores were well below 25\% for questions 17, 18, and 19 in both student groups. This unexpected finding may potentially be attributed to the possibility that the concepts represented in these questions were insufficiently covered in both curricula and therefore, represented a significant challenge for students in both groups.

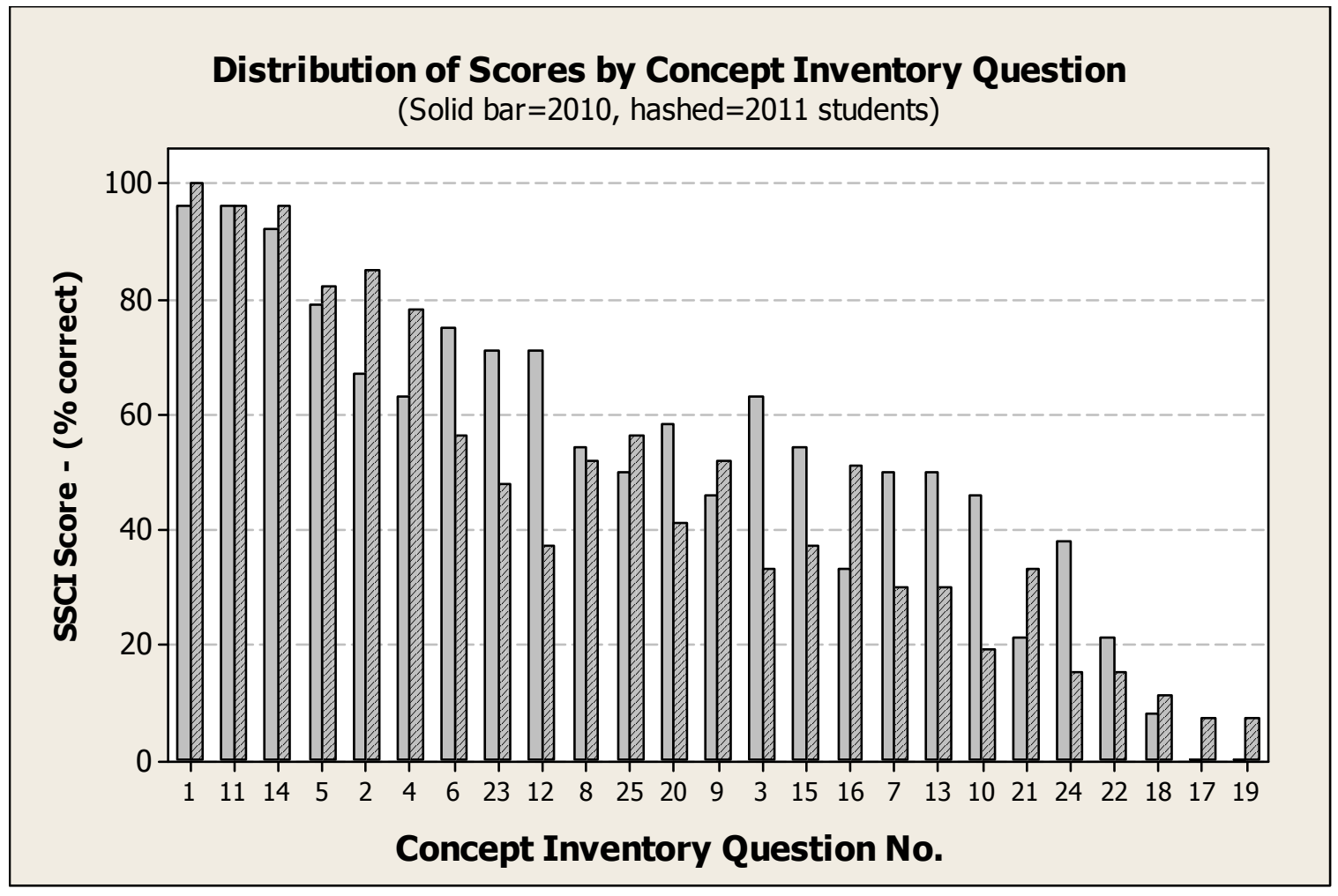

Figure 3: SSCI assessment performance for BME students who did (solid) and did not (hashed) participate in the cross-disciplinary laboratories. Each bar represents the SSCI score computed as the percentage of students who responded correctly to a given question. 


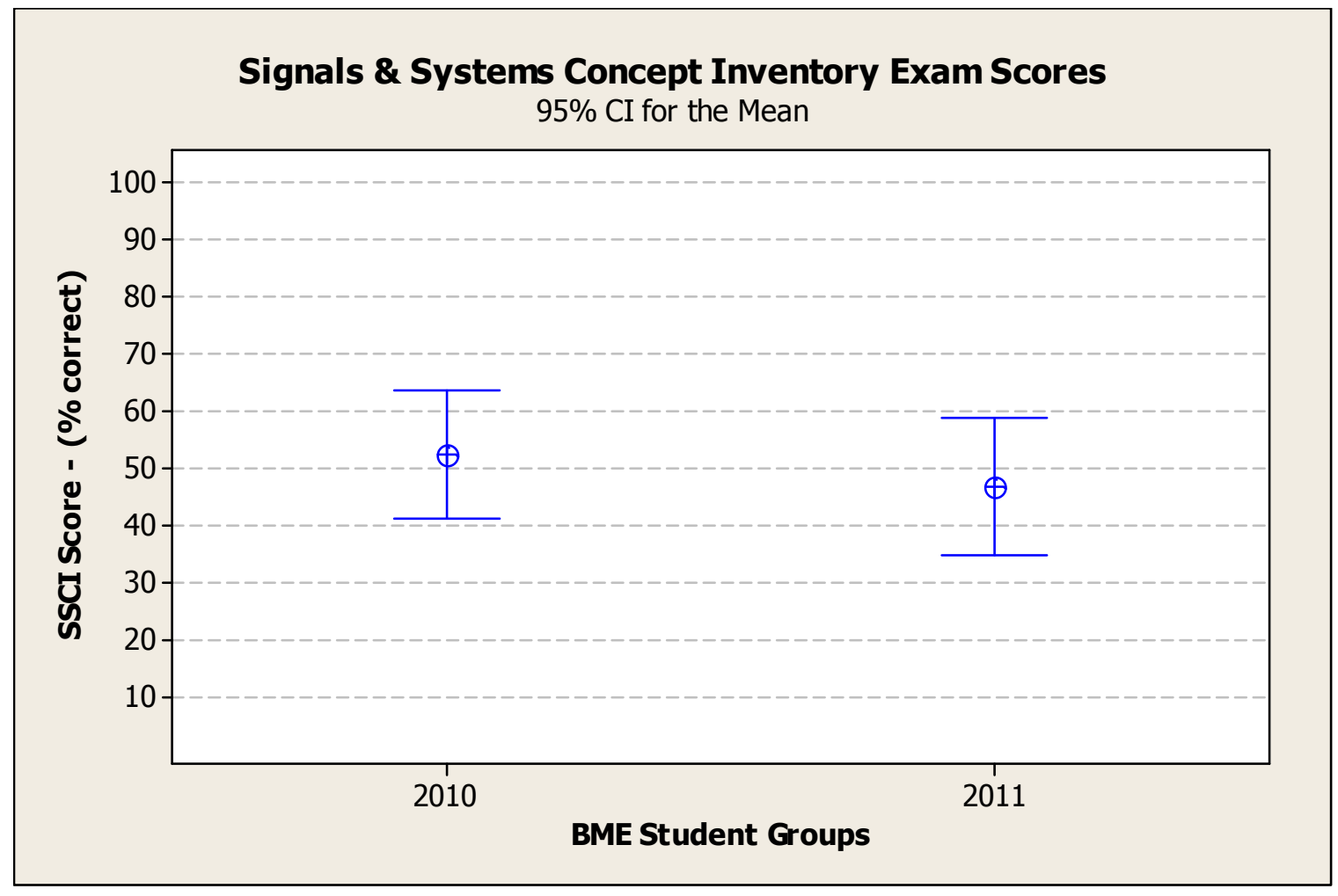

Figure 4: Overall SSCI assessment performance for BME students who did not participate in the cross-disciplinary laboratories (2010) and for those who did (2011). Each interval plot represents an average SSCI score computed across all questions and all students in the given group. No significant $(\mathrm{p}>0.05)$ difference in performance was found between the two student groups.

\section{Conclusion/Future Work}

It is concluded that students performed similarly on the SSCI assessment exam despite the changes in the curriculum. Specifically, students in the new curriculum experienced $25 \%$ fewer lecture hours in the core discipline of signals and system, but they took a cross-disciplinary laboratory that combined Biosystems and Physiology in place of the previous Physiology laboratory. The faculty is pleased that there was no loss in student comprehension in signals and systems in the new curriculum.

Anecdotal comments from students suggest some enhancement in the students' ability to solve multi-disciplinary problems. However, future work to complete the formal assessment of the effectiveness of the new approach will test student knowledge in the other core discipline, physiology, as well as in the integration of the two disciplines. As no proven concept inventories have been developed for these subjects, the faculty will develop methods that they expect to be reliable methods to assess student knowledge in these areas. 
The faculty members acknowledge that the scheduling of the SSCI was not identical for the two groups. Logistical complexities prohibited the tests from being administered after the same amount of time following the courses for students in both curricula. While more time had elapsed for students in the pevioius curriculum, they may also have had some concepts reinforced in other classes and in their senior design project during this intervening time. To address the shortcomings of the study arising from different testing schedules, the faculty intends to administer follow-up SSCI exams to the cohort in the new curriculum. In addition, the faculty will develop new assessment tools, such as surveys and interviews, that will allow a triangulation approach to be taken to the assessment process.

An unintended benefit of the use of the SSCI has been the revelation of concepts in which the students have apparent misperceptions. The faculty plan to use this knowledge, as well as similar insights from future assessments, to guide improvements in how certain topics are covered in both the lecture and laboratory.

[1] Criteria For Accrediting Engineering Programs, Effective for Evaluations During the 2010-2011 Accreditation Cycle, Engineering Accreditation Commission, ABET, Inc., www.abet.org

[2] National Academy of Engineering, "The Engineer of 2020: Visions of Engineering in the New Century," The National Academies Press, 2004.

[3] Buck, J.R.; Wage, K.E.; Hjalmarson, M.A.; Nelson, J.K.; , "Comparing student understanding of signals and systems using a concept inventory, a traditional exam and interviews," Frontiers In Education Conference Global Engineering: Knowledge Without Borders, Opportunities Without Passports, 2007. FIE '07. 37th Annual, vol., no., pp.S1G-1-S1G-6, 10-13 Oct. 2007

[4] Nasr, R.; Hall, S.R.; Garik, P.; , "Understanding Naive Reasonings in Signals and Systems: A Foundation for Designing Effective Instructional Material," Digital Signal Processing Workshop and 5th IEEE Signal Processing Education Workshop, 2009. DSP/SPE 2009. IEEE 13th, vol., no., pp.720-725, 4-7 Jan. 2009

[5] Wage, K.E.; Buck, J.R.; Welch, T.B.; Wright, C.H.G.; , "The continuous-time signals and systems concept inventory," Acoustics, Speech, and Signal Processing, 2002. Proceedings. (ICASSP '02). IEEE International Conference, vol.4, pp. IV-4112- IV-4115 vol.4, 2002

[6] Buck, J. R., Wage, K. E., Hjalmarson, M. A., Nelson, J. K., “Comparing Student Understanding of Signals and Systems Using a Concept Inventory, a Traditional Exam and Interviews", . Proceedings of the 37th ASEE/IEEE Frontiers in Education Conference, pp. S1G1-S1G6, 2007 